\title{
A Bayesian Technique for Real and Integer Parameters Estimation in Linear Models and Its Application to GNSS High Precision Positioning
}

\author{
Javier G. Garcia, Member, IEEE, Pedro A. Roncagliolo, Member, IEEE, and
} Carlos H. Muravchik, Senior Member, IEEE

\begin{abstract}
A novel Bayesian technique for the joint estimation of real and integer parameters in a linear measurement model is presented. The integer parameters take values on a finite set, and the real ones are assumed to be a Gaussian random vector. The posterior distribution of these parameters is sequentially determined as new measurements are incorporated. This is a mixed distribution with a Gaussian continuous part and a discrete one. Estimators for the integer and real parameters are derived from this posterior distribution. A Maximum A Posteriori (MAP) estimator modified with the addition of a confidence threshold is used for the integer part and a Minimum Mean Squared Error (MMSE) is used for the real parameters. Two different cases are addressed: i) both real and integer parameters are time invariant and ii) the integer parameters are time invariant but the real ones are time varying. Our technique is applied to the GNSS carrier phase ambiguity resolution problem, that is key for high precision positioning applications. The good performance of the proposed technique is illustrated through simulations in different scenarios where different kind of measurements as well as different satellite visibility conditions are considered. Comparisons with state-of-the-art ambiguity solving algorithms confirm performance improvement. The new method is shown to be useful not only in the estimation stage but also for validating the estimates ensuring a predefined success rate through proper threshold selection.
\end{abstract}

Index Terms-Bayesian estimation, carrier phase ambiguity resolution, GNSS, integer parameter estimation.

\section{INTRODUCTION}

$\mathbf{T}$ HE joint estimation of integer and real parameters is a problem that is present in an important number of current applications such as Magnetic Resonance Imaging (MRI), Interferometric Synthetic Aperture Radar (InSAR), Communications (especially for multi-input/multi-output channels),

Manuscript received February 17, 2015; revised July 27, 2015; accepted October 16, 2015. Date of publication November 12, 2015; The associate editor coordinating the review of this manuscript and approving it for publication was Dr. Pascal Larzabal. This work was funded by the Argentine ANPCyT, grant PICT 2011-0909, Universidad Nacional de La Plata grant 11-I-166, and CIC Pcia de Buenos Aires.

The authors are with the Instituto de Investigaciones en Electrónica, Control y Procesamiento de Señales LEICI, Facultad de Ingeniería, Universidad Nacional de La Plata (UNLP), Argentina.

Color versions of one or more of the figures in this paper are available online at http://ieeexplore.ieee.org.

Digital Object Identifier 10.1109/TSP.2015.2500195
Acoustic Interferometry, Cryptography, etc [1], [2]. However, in the last couple of decades much of the emphasis went to the Global Navigation Satellite Systems (GNSS) Carrier Phase Ambiguity Resolution. That is the determination of the unknown integer number of carrier cycles in the phase measurements. Once these integer values are found, carrier phase information can be used as very precise range measurements. This is the basis of high precision GNSS positioning and it applies to a great variety of GNSS applications such as navigation, attitude determination, surveying, geodesy, and geophysics.

Typically, the ambiguity resolution involves two different steps i) reduction and ii) search. The reduction stage deals with the generation of the set of potential integer candidates. This set should be big enough to ensure that the correct integer vector belongs to it and small enough to allow for a fast search stage. Two typical reduction techniques are employed in practice, the Korkine-Zolotareff (KZ) [3] reduction and the Lenstra-Lenstra-Lovasz (LLL) reduction [4]. Due to the existence of a polynomial time algorithm for its computation, the LLL reduction is more widely used in practice. On the other hand, the search stage focuses on the selection of the best candidate among all possible ones. To this end, typically the Integer Least Squares criterion is employed. However, this criterion makes it difficult to incorporate prior information as well as to sequentially compute estimates. In addition, in the case of little or scarce measurements its success rate tends to be poor.

Most of ambiguity resolution methods discussed in the literature are based in a coarse estimation followed by a refinement process to find the final estimate [5]. In the case of the Ambiguity Function Method, the possible integer values are restricted to a grid corresponding to a discretization of the position variables [6]. In the Least Squares Ambiguity Search method, a floating solution that does not take into account the discrete nature of the ambiguities is found first minimizing the mean squared error. Then, the final result, known as fixed solution, is searched for in the surroundings of the floating solution [7]. The size of the search space depends on the covariance matrix of the floating solution and the number of ambiguities to be estimated. The LAMBDA (for Least-squares AMBiguity Decorrelation Approach) method uses a linear integer transformation to make the covariance matrix near diagonal. This transformation notably reduces the search space allowing a more efficient search process. In [2], techniques based in the LLL algorithm 
[8] are proposed for the problem of estimation and verification of real and integer parameters. The class of integer equivariant (IE) estimators, enclosing integer (I) as well as linear unbiased estimators, and the Best (i.e., unbiased and minimum variance in their class) Integer Equivariant (BIE) estimator are presented in [9]. A unified framework, called integer aperture (IA), for estimating and validating the GNSS ambiguity solution is introduced in [10] and expanded in [11]. Based on the IA theory, the known ratio test used for validating the ambiguities solution was revisited in [12]. Finally, the Bayesian approach was employed in the context of GNSS ambiguity resolution in [13]-[17]. [13]-[16] yield a posterior distribution for all variables, discrete and continuous, conditional to the observed quantities; [16] determines confidence regions for the baseline estimation based on GPS measurements; and [17] presents several well known Bayesian estimation schemes and applies them to the ambiguity resolution problem.

Very often, the proposed ambiguity resolution methods deal with the problem of differential positioning, where the difference of the position of two antennas (with its associated receivers) is estimated. Usually, one of the receivers acts as a reference receiver whereas the other is called the rover. The vector formed by the difference of position from the rover to the reference is known as the baseline. When both receivers do not move, the baseline is called static. Conversely, when at least one of the receivers moves, the baseline is called kinematic. Typically, ambiguity resolution techniques are thought for static baselines, but in a kinematic scenario they often become relevant, when the best accuracy is sought for. Actually, in a kinematic scenario a new set of baseline Cartesian components is to be estimated for each measurement epoch, making the ambiguity resolution process more difficult. However, there are some techniques that use the baseline movement to improve the success of the ambiguity determination.

As opposed to the classical approach where the parameters to be estimated are seen as deterministic, in the Bayesian approach they are considered as random variables. The key of this approach is to start with a prior distribution of the parameters, and then to incorporate the knowledge of the available measurements to form the posterior distribution. Based on this distribution several estimators can be obtained, like the MAP and the MMSE [18]. One of the best known Bayesian estimators is the Kalman filter (KF), which is the optimal Bayesian estimator for a parameter and measurement linear model, with additive Gaussian noises. The KF is usually implemented sequentially, i.e., the estimated values are updated as new measurements become available. Unfortunately, since all the distributions involved are continuous -Gaussian in fact-, the KF can not be directly applied to the estimation of integer parameters while retaining its optimality.

In this work, whose main idea was exposed in [19], we introduce a novel technique to jointly estimate real and integer parameters in linear models. It is based (i) in the Bayesian estimation philosophy and (ii) on the assumption that the integer parameters belong to a finite set that it is known in advance, while the real ones come from a realization of a Gaussian random vector. The basis of the method is to sequentially determine the posterior distribution of the parameters to be estimated as new measurements are incorporated. This posterior distribution is a mixed one, with a Gaussian continuous part and a discrete part that accounts for the probability of each of the elements of the finite set. Estimators for the integer and real parameters are derived from the posterior distribution. For the integer part, it is natural to use a MAP estimator because a MMSE estimator could give non-integer estimates. For the real paramters, a MMSE estimator is used. In order to get a reliable estimation, a confidence threshold is used in the discrete part so that the method provides estimates only when they are good enough, as it will be explained in Sections III-C and VII. The Bayesian approach was already used in [13]-[15], our technique is substantially different in several ways such as the optimality criterion, the hypotheses used for the integer parameters and the use of a sequential implementation. [17] applies several Bayesian models to the ambiguity resolution problem but it remains in a general context, somewhat far from practice. Although our technique is primarily devoted to the estimation of static parameters, it is extended to time-varying real parameters (e.g., as in the case of kinematic baselines in GNSS differential positioning) following a linear dynamical model. None of the previously mentioned Bayesian ambiguity resolution techniques are directly prepared to do that. In this paper, we mainly focus on the search stage and we provide an example on how to deal with the reduction stage.

The work is organized as follows. In Section II, Bayesian estimation is shortly reviewed. The new method is introduced in Section III. In Section IV, the method is extended to timevarying real parameters. A comparison with other sequential techniques based on the Bayesian approach and a brief analysis of the computational efficiency of our procedure is done in Section V. Then, in Section VI, this technique is applied to the GNSS carrier phase ambiguity resolution problem for high precision applications. The performance of the proposed algorithm is evaluated via simulations and compared to the LAMBDA method in Section VII. Finally, Section VIII presents the conclusions and describes further work.

\section{THE BAYESIAN APPROACH}

The Bayesian approach was preferred because it permits to include easily prior information in the estimators [20], [21].

If $\mathbf{x}$ is the vector of parameters to be estimated and $\mathbf{y}$ is the measurements vector, the posterior distribution $p(\mathbf{x} \mid \mathbf{y})$ provides all the information about $\mathbf{x}$ given the measurements $\mathbf{y}$. Bayes' theorem gives an expression of the posterior distribution $p(\mathbf{x} \mid \mathbf{y})$ in terms of the prior distribution, $p(\mathbf{x})$, and the likelihood function, $p(\mathbf{y} \mid \mathbf{x})$,

$$
p(\mathbf{x} \mid \mathbf{y})=\frac{p(\mathbf{y} \mid \mathbf{x}) p(\mathbf{x})}{\int p(\mathbf{y} \mid \mathbf{x}) p(\mathbf{x}) d \mathbf{x}} .
$$

From this posterior distribution, several estimators can be proposed depending on the desired optimality criteria [18].

Starting from the Bayes' theorem (1), a formula for the sequential computation of the posterior distribution can be obtained. Let $\mathbf{y}_{k}$ be the measurement vector corresponding to time $t_{k}$ and $\mathbf{y}_{1: k}$ be the set of all available measurements until $t_{k}$, i.e., $\mathbf{y}_{1: k}=\left[\mathbf{y}_{k}^{T}, \mathbf{y}_{k-1}^{T}, \ldots, \mathbf{y}_{1}^{T}\right]^{T}$. It is useful to have an expression 
to update the posterior distribution each time a new measurement is available. Assuming that $p\left(\mathbf{y}_{k} \mid \mathbf{x}, \mathbf{y}_{1: k-1}\right)=p\left(\mathbf{y}_{k} \mid \mathbf{x}\right)$, applying some conditional distribution's properties and with some algebraic work (see e.g. [20] chapter 2) it can be shown that

$$
p\left(\mathbf{x} \mid \mathbf{y}_{1: k}\right)=\frac{p\left(\mathbf{y}_{k} \mid \mathbf{x}\right) p\left(\mathbf{x} \mid \mathbf{y}_{1: k-1}\right)}{p\left(\mathbf{y}_{k} \mid \mathbf{y}_{1: k-1}\right)}
$$

where $p\left(\mathbf{y}_{k} \mid \mathbf{y}_{1: k-1}\right)$ is calculated as

$$
p\left(\mathbf{y}_{k} \mid \mathbf{y}_{1: k-1}\right)=\int p\left(\mathbf{y}_{k} \mid \mathbf{x}\right) p\left(\mathbf{x} \mid \mathbf{y}_{1: k-1}\right) d \mathbf{x}
$$

In this way, an explicit expression for the sequential calculation of the posterior distribution is obtained. We start with $p\left(\mathbf{x} \mid \mathbf{y}_{1}\right)$; once we have the following measurement $\mathbf{y}_{2}$, we can calculate $p\left(\mathbf{x} \mid \mathbf{y}_{1: 2}\right)$; with $\mathbf{y}_{3}, p\left(\mathbf{x} \mid \mathbf{y}_{1: 3}\right)$; and so on.

\section{PRoposed TeChNiQue}

\section{A. Measurements Model}

We assume that the parameters to be estimated, $\mathbf{x} \in \mathbb{R}^{p}$ (reals) and $\mathbf{z} \in \mathbb{Z}^{q}$ (integers), are related to the measurements at time $t_{k}, \mathbf{y}_{k} \in \mathbb{R}^{m}$, through the following model

$$
\mathbf{y}_{k}=\mathbf{A}_{k} \mathbf{x}+\mathbf{B}_{k} \mathbf{z}+\mathbf{v}_{k}
$$

where matrices $\mathbf{A}_{k} \in \mathbb{R}^{m \times p}$ and $\mathbf{B}_{k} \in \mathbb{R}^{m \times q}$ are known full column rank, and $\mathbf{v}_{k}$ is a zero mean white Gaussian noise vector with covariance matrix $\Sigma_{\mathbf{v}, k}$. The integer parameter $\mathbf{z}$ belongs to a finite set with $N_{T}$ elements, i.e., the true value of $\mathbf{z}$ is some $\mathbf{z}^{i}$ where $i=1, \ldots, N_{T}$. A technique for the generation of this set is given in Appendix. In addition, it is assumed that $\mathbf{x}$ and $\mathbf{z}$ remain constant during the observation time. In order to apply the Bayesian approach to this problem, the posterior distribution of $\mathbf{x}$ and $\mathbf{z}$ given the measurements until time $t_{k}$ must be obtained. Then, similarly to (2), we find that

$$
p\left(\mathbf{x}, \mathbf{z} \mid \mathbf{y}_{1: k}\right)=\frac{p\left(\mathbf{y}_{k} \mid \mathbf{x}, \mathbf{z}\right) p\left(\mathbf{x}, \mathbf{z} \mid \mathbf{y}_{1: k-1}\right)}{p\left(\mathbf{y}_{k} \mid \mathbf{y}_{1: k-1}\right)}
$$

The concept is to start with a weakly informative prior distribution of $\mathbf{x}$ and $\mathbf{z}, p(\mathbf{x}, \mathbf{z})$, and then adjust it as new measurements $\mathbf{y}_{1}, \ldots, \mathbf{y}_{k}$, are incorporated, leading to $p\left(\mathbf{x}, \mathbf{z} \mid \mathbf{y}_{1: k}\right)$.

\section{B. Sequential Computation of the Posterior Distribution}

The method aims to obtain an explicit formula to sequentially compute $p\left(\mathbf{x}, \mathbf{z} \mid \mathbf{y}_{1: k}\right)$. For this purpose, the prior distribution of the continuous variables $\mathbf{x}$, as well as of the discrete ones $\mathbf{z}$, must be known. Given the different nature of the random variables, the result is a mixed continuous/discrete distribution. Assuming that the prior distributions of $\mathbf{x}$ and $\mathbf{z}$ are independent, then

$$
p(\mathbf{x}, \mathbf{z})=p(\mathbf{x}) p(\mathbf{z}) .
$$

We also consider a Gaussian prior distribution of $\mathbf{x}$ with mean $\boldsymbol{\mu}_{\mathbf{x}, 0}$ and covariance $\boldsymbol{\Sigma}_{\mathbf{x}, 0}$, denoted

$$
p(\mathbf{x})=\mathcal{N}\left(\mathbf{x} ; \boldsymbol{\mu}_{\mathbf{x}, 0}, \boldsymbol{\Sigma}_{\mathbf{x}, 0}\right)
$$

and given by

$$
\mathcal{N}(\mathbf{x} ; \boldsymbol{\mu}, \boldsymbol{\Sigma})=\frac{\exp \left\{-\frac{1}{2}(\mathbf{x}-\boldsymbol{\mu})^{T} \boldsymbol{\Sigma}^{-1}(\mathbf{x}-\boldsymbol{\mu})\right\}}{(2 \pi)^{\frac{n}{2}}|\boldsymbol{\Sigma}|^{\frac{1}{2}}} .
$$

The prior distribution of $\mathbf{z}$ is assumed to have the following structure

$$
p(\mathbf{z})=\sum_{i=1}^{N_{T}} w_{0}^{i} \delta\left(\mathbf{z}-\mathbf{z}^{i}\right)
$$

where $w_{0}^{i}=P\left\{\mathbf{z}=\mathbf{z}^{i}\right\}$ is the probability that $\mathbf{z}$ equals $\mathbf{z}^{i}$. Therefore, from (6), (7) and (9) the joint prior distribution of $\mathbf{x}$ and $\mathbf{z}$ can be expressed as

$$
p(\mathbf{x}, \mathbf{z})=\sum_{i=1}^{N_{T}} w_{0}^{i} \mathcal{N}\left(\mathbf{x} ; \boldsymbol{\mu}_{\mathbf{x}, 0}, \boldsymbol{\Sigma}_{\mathbf{x}, 0}\right) \delta\left(\mathbf{z}-\mathbf{z}^{i}\right) .
$$

Hence, fixing the values of $\boldsymbol{\mu}_{\mathbf{x}, 0}, \quad \boldsymbol{\Sigma}_{\mathbf{x}, 0}$ and $w_{0}^{i}$ with $i=1, \ldots, N_{T}$, completely defines $p(\mathbf{x}, \mathbf{z})$. These values depend on the prior information about the parameters to be estimated. If there is no or little prior information, a weakly informative distribution should be used. For $p(\mathbf{z})$, a non-informative distribution is the uniform $w_{0}^{i}=1 / N_{T}$. Since $p(\mathbf{x})$ is Gaussian, it should have a mean as close as possible to the value to be estimated, and a large enough $\Sigma_{\mathbf{x}, 0}$ to account for all possible errors. A convenient choice for the covariance is $\Sigma_{\mathbf{x}, 0}=\sigma_{x_{0}}^{2} I_{p}$, where $\sigma_{x_{0}}^{2}$ is a constant selected according to the preceding remark, and $I_{p}$ is the $p \times p$ identity matrix. From (5) it can be seen that, both the likelihood function $p\left(\mathbf{y}_{1} \mid \mathbf{x}, \mathbf{z}\right)$ and the prior distribution $p(\mathbf{x}, \mathbf{z})$ are needed to obtain the first posterior distribution. From (4) it follows that

$$
p\left(\mathbf{y}_{1} \mid \mathbf{x}, \mathbf{z}\right)=\mathcal{N}\left(\mathbf{y}_{1} ; \mathbf{A}_{1} \mathbf{x}+\mathbf{B}_{1} \mathbf{z}, \boldsymbol{\Sigma}_{\mathbf{v}, 1}\right),
$$

and the numerator of (5) for $k=1$ results

$$
\begin{array}{r}
p\left(\mathbf{y}_{1} \mid \mathbf{x}, \mathbf{z}\right) p(\mathbf{x}, \mathbf{z})=\sum_{i=1}^{N_{T}} w_{0}^{i} \mathcal{N}\left(\mathbf{y}_{1} ; \mathbf{A}_{1} \mathbf{x}+\mathbf{B}_{1} \mathbf{z}, \boldsymbol{\Sigma}_{\mathbf{v}, 1}\right) \\
\times \mathcal{N}\left(\mathbf{x} ; \boldsymbol{\mu}_{\mathbf{x}, 0}, \boldsymbol{\Sigma}_{\mathbf{x}, 0}\right) \delta\left(\mathbf{z}-\mathbf{z}^{i}\right) .
\end{array}
$$

After a tedious algebraic manipulation of (12), similar to the development in [22], and using (5), we get

$$
p\left(\mathbf{x}, \mathbf{z} \mid \mathbf{y}_{1}\right)=\sum_{i=1}^{N_{T}} \mathcal{N}\left(\mathbf{x} ; \boldsymbol{\mu}_{\mathbf{x}, 1}^{i}, \boldsymbol{\Sigma}_{\mathbf{x}, 1}\right) w_{1}^{i} \delta\left(\mathbf{z}-\mathbf{z}^{i}\right)
$$


where

$$
\begin{aligned}
\boldsymbol{\mu}_{\mathbf{x}, 1}^{i} & =\boldsymbol{\mu}_{\mathbf{x}, 0}+\sigma_{x_{0}}^{2} \mathbf{A}_{1}^{T} \mathbf{\Sigma}_{\mathbf{y}, 1}^{-1}\left(\mathbf{y}_{1}-\boldsymbol{\mu}_{\mathbf{y}, 1}^{i}\right), \\
\boldsymbol{\Sigma}_{\mathbf{x}, 1} & =\sigma_{x_{0}}^{2} I_{p}+\sigma_{x_{0}}^{4} \mathbf{A}_{1}^{T} \boldsymbol{\Sigma}_{\mathbf{y}, 1}^{-1} \mathbf{A}_{1}, \\
\boldsymbol{\mu}_{\mathbf{y}, 1}^{i} & =\mathbf{A}_{1} \boldsymbol{\mu}_{\mathbf{x}, 0}+\mathbf{B}_{1} \mathbf{z}^{i}, \\
\boldsymbol{\Sigma}_{\mathbf{y}, 1} & =\sigma_{x_{0}}^{2} \mathbf{A}_{1} \mathbf{A}_{1}^{T}+\boldsymbol{\Sigma}_{\mathbf{v}, 1} \text { and } \\
w_{1}^{i} & =\frac{w_{0}^{i} \mathcal{N}\left(\mathbf{y}_{1} ; \boldsymbol{\mu}_{\mathbf{y}, 1}^{i}, \boldsymbol{\Sigma}_{\mathbf{y}, 1}\right)}{\sum_{i=1}^{N_{T}} w_{0}^{i} \mathcal{N}\left(\mathbf{y}_{1} ; \boldsymbol{\mu}_{\mathbf{y}, 1}^{i}, \mathbf{\Sigma}_{\mathbf{y}, 1}\right)} .
\end{aligned}
$$

In the above expressions we assumed that $\Sigma_{\mathbf{x}, 0}=\sigma_{x_{0}}^{2} I_{p}$, for other choices of $\Sigma_{\mathbf{x}, 0}$ minor changes are needed (see the general expression that follows). After $p\left(\mathbf{x}, \mathbf{z} \mid \mathbf{y}_{1}\right)$ has been obtained, and the second measurement $\mathbf{y}_{2}$ is available, $p\left(\mathbf{x}, \mathbf{z} \mid \mathbf{y}_{1: 2}\right)$ is found in the same way.

In general, if the distribution $p\left(\mathbf{x}, \mathbf{z} \mid \mathbf{y}_{1: k-1}\right)$ is

$$
\begin{aligned}
p\left(\mathbf{x}, \mathbf{z} \mid \mathbf{y}_{1: k-1}\right) & \\
& =\sum_{i=1}^{N_{T}} \mathcal{N}\left(\mathbf{x} ; \boldsymbol{\mu}_{\mathbf{x}, k-1}^{i}, \boldsymbol{\Sigma}_{\mathbf{x}, k-1}\right) w_{k-1}^{i} \delta\left(\mathbf{z}-\mathbf{z}^{i}\right)
\end{aligned}
$$

the posterior distribution $p\left(\mathbf{x}, \mathbf{z} \mid \mathbf{y}_{1: k}\right)$ becomes

$$
p\left(\mathbf{x}, \mathbf{z} \mid \mathbf{y}_{1: k}\right)=\sum_{i=1}^{N_{T}} \mathcal{N}\left(\mathbf{x} ; \boldsymbol{\mu}_{\mathbf{x}, k}^{i}, \boldsymbol{\Sigma}_{\mathbf{x}, k}\right) w_{k}^{i} \delta\left(\mathbf{z}-\mathbf{z}^{i}\right)
$$

where

$$
\begin{aligned}
\boldsymbol{\mu}_{\mathbf{x}, k}^{i} & =\boldsymbol{\mu}_{\mathbf{x}, k-1}^{i}+\boldsymbol{\Sigma}_{\mathbf{x}, k-1} \mathbf{A}_{k}^{T} \boldsymbol{\Sigma}_{\mathbf{y}, k}^{-1}\left(\mathbf{y}_{k}-\boldsymbol{\mu}_{\mathbf{y}, k}^{i}\right) \\
\boldsymbol{\Sigma}_{\mathbf{x}, k} & =\boldsymbol{\Sigma}_{\mathbf{x}, k-1}+\boldsymbol{\Sigma}_{\mathbf{x}, k-1} \mathbf{A}_{k}^{T} \boldsymbol{\Sigma}_{\mathbf{y}, k}^{-1} \mathbf{A}_{k} \boldsymbol{\Sigma}_{\mathbf{x}, k-1} \\
\boldsymbol{\mu}_{\mathbf{y}, k}^{i} & =\mathbf{A}_{k} \boldsymbol{\mu}_{\mathbf{x}, k-1}^{i}+\mathbf{B}_{k} \mathbf{z}^{i} \\
\boldsymbol{\Sigma}_{\mathbf{y}, k} & =\mathbf{A}_{k} \boldsymbol{\Sigma}_{\mathbf{x}, k-1} \mathbf{A}_{k}^{T}+\boldsymbol{\Sigma}_{\mathbf{v}, k} \text { and } \\
w_{k}^{i} & =\frac{w_{k-1}^{i} \mathcal{N}\left(\mathbf{y}_{k} ; \boldsymbol{\mu}_{\mathbf{y}, k}^{i}, \boldsymbol{\Sigma}_{\mathbf{y}, k}\right)}{\sum_{i=1}^{N_{T}} w_{k-1}^{i} \mathcal{N}\left(\mathbf{y}_{k} ; \boldsymbol{\mu}_{\mathbf{y}, k}^{i}, \boldsymbol{\Sigma}_{\mathbf{y}, k}\right)}
\end{aligned}
$$

It can be seen that distributions such as (19) are conjugate priors of distributions such as (20) hence, the posterior (20) repeats the structure of the a-priori [18]. Notice that (20) can be factored as $p\left(\mathbf{x}, \mathbf{z} \mid \mathbf{y}_{1: k}\right)=p\left(\mathbf{x} \mid \mathbf{z}, \mathbf{y}_{1: k}\right) p\left(\mathbf{z} \mid \mathbf{y}_{1: k}\right)$, with $p\left(\mathbf{x} \mid \mathbf{z}, \mathbf{y}_{1: k}\right)=$ $\mathcal{N}\left(\mathbf{x} ; \boldsymbol{\mu}_{\mathbf{x}, k}^{i}, \mathbf{\Sigma}_{\mathbf{x}, k}\right)$ and $p\left(\mathbf{z} \mid \mathbf{y}_{1: k}\right)=\sum_{i=1}^{N_{T}} w_{k}^{i} \delta\left(\mathbf{z}-\mathbf{z}^{i}\right)$.

\section{Derivation of the Estimators}

According to the previous considerations, the posterior distribution $p\left(\mathbf{x}, \mathbf{z} \mid \mathbf{y}_{1: k}\right)$ conveys all the information about the parameters to be estimated, $\mathbf{x}$ and $\mathbf{z}$, in the measurements $\mathbf{y}_{1: k}$. In order to select the best Bayesian estimators some optimality criteria must be adopted. For the case of $\mathbf{z}$, it seems natural to use a MAP estimator, since it guarantees integer estimates as desired. For the real variables estimator, once the integer $\mathbf{z}$ is chosen, both the MAP and MMSE criteria give the same estimator, the conditional mean (or equivalently the mean of the posterior distribution). Therefore, the selected estimators of $\mathbf{x}$ and $\mathbf{z}$ are

$$
\begin{aligned}
\hat{\mathbf{z}}_{k} & =\mathbf{z}^{J} \mid J=\arg \max _{i=1, \ldots, N_{T}} w_{k}^{i} \quad \text { and } \\
\hat{\mathbf{x}}_{k} & =\boldsymbol{\mu}_{\mathbf{x}, k}^{J}
\end{aligned}
$$

With these expressions estimates for $\mathbf{z}$ and $\mathbf{x}$ are obtained for any $k$, however, their reliability could be low at an early stage. In order to address this aspect, we propose the addition of a threshold to the integer parameters estimator. That is, estimates are considered valid only if it holds that $w_{k}^{J}>\gamma$, with $\gamma$ a constant threshold to be defined. With this strategy, we will have valid estimates only when the reliability condition is met.

The value of $\gamma$ can be appropriately selected by analyzing the meaning of $w_{k}^{i}$. From (20) it follows that $w_{k}^{i}=P\{\mathbf{z}=$ $\left.\mathbf{z}^{i} \mid \mathbf{y}_{1: k}\right\}$. Hence, if a high degree of certainty is required, $\gamma$ should be chosen close to 1 . Of course, the closer $\gamma$ is to 1 the larger is the number of measurements required to obtain a valid estimate. Then, the balance between reliability and speed is controlled by the choice of $\gamma$. In Section VII, it will be seen through numerical simulations that $\gamma$ determines a lower bound for the Success Rate SR (i.e., the number of correct integer estimates over the number of trials) of the estimation method.

\section{Operation of the Method}

In order to show the behavior of the developed method in a qualitative way, let's consider the simplified problem of 1D positioning using dual frequency, sinusoidal beacons similar to that of Sec. VII-A in [2]. This very simplified example is the basis of most electromagnetic distance measurement (EDM) equipment used by surveyors and geodesists [23]. The objective is to estimate the unknown receiver position $x_{R} \in \mathbb{R}$ using two sinusoidal signals of different frequencies sent by a beacon (transmitter) located at a known position $x_{T}$. Assume a receiver (synchronized with the transmitter) that measures the phase of the two signals emitted by the beacon. Due to the periodic nature of the transmitted signal, the receiver is only able to measure these phases modulo some integer multiple of the wavelengths. Let $y_{1}$ and $y_{2}$ be the measured phases. It is straightforward to get that (see Fig. 1)

$$
x_{R}=x_{T}+\lambda_{1} z_{1}+y_{1}, \quad x_{R}=x_{T}+\lambda_{2} z_{2}+y_{2}
$$

where $\lambda_{1}$ and $\lambda_{2}$ are the wavelengths of the transmitted signals. Equivalently, (28) in matrix form results in

$$
\left[\begin{array}{l}
y_{1}+x_{T} \\
y_{2}+x_{T}
\end{array}\right]=\left[\begin{array}{l}
1 \\
1
\end{array}\right] x_{R}-\left[\begin{array}{cc}
\lambda_{1} & 0 \\
0 & \lambda_{2}
\end{array}\right]\left[\begin{array}{l}
z_{1} \\
z_{2}
\end{array}\right]+\left[\begin{array}{l}
v_{1} \\
v_{2}
\end{array}\right]
$$

where an additional unavoidable noise term (i.e., $v_{1}$ and $v_{2}$ ) was added. It is easily seen from (29) that to solve this problem, the estimation of one real parameter $x_{R}$ and two integer parameters $z_{1}$ and $z_{2}$ are needed. In the rest of this section, we use our method to illustrate how it works. A test scenario with measurements from different instants was generated. The real position was set as $x_{R}=10 \mathrm{~m}$, the signal wavelengths were chosen as $\lambda_{1}=1.075 \mathrm{~m}$ and $\lambda_{2}=0.925 \mathrm{~m}, 20$ seconds of 
GARCIA et al: : A BAYESIAN TECHNIQUE FOR REAL AND INTEGER PARAMETERS ESTIMATION IN LINEAR MODELS AND ITS APPLICATION TO GNSS HIGH PRECISION POSITIONING

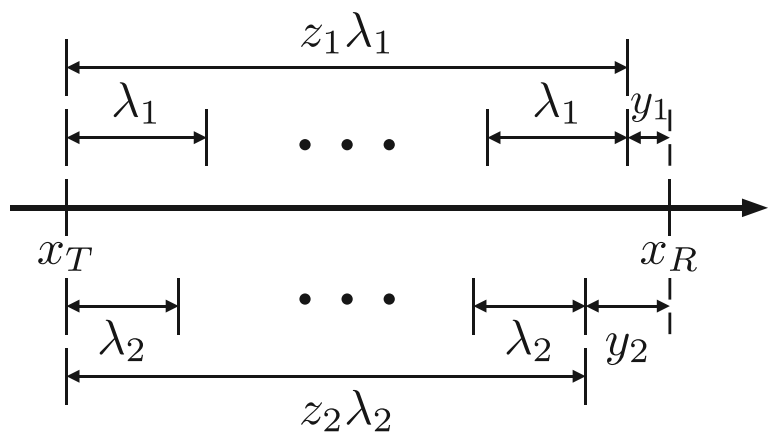

Fig. 1. 1D positioning using a dual frequency beacon: $x_{T}$ is the beacon position, $x_{R}$ is the receiver position, $\lambda_{1}$ and $\lambda_{2}$ are the wavelengths of the transmitted signals, $z_{1}$ and $z_{2}$ are the integer number of wavelengths, whereas $y_{1}$ and $y_{2}$ are the measured phase of each signal at the receiver.

data were generated with a sampling period of $1 \mathrm{sec}$. For simplicity, the transmitter position was set to $x_{T}=0 \mathrm{~m}$. The set of possible integers were generated to ensure that the true value belongs to the generated set with a high probability. Thus the range of values for $z_{i}$ was $\left(\operatorname{round}\left(\bar{z}_{i}-3 \sigma_{\bar{z}_{i}}\right)\right.$, round $\left.\left(\bar{z}_{i}+3 \sigma_{\bar{z}_{i}}\right)\right)$ with $\bar{z}_{i}=\left(y_{i}-\mu_{x, 0}\right) / \lambda_{i}$ and $\sigma_{\bar{z}_{i}}=\left(1 / \lambda_{i}\right) \sqrt{\sigma_{v}^{2}+\sigma_{x, 0}^{2}}$ for $i=1,2$. Zero mean Gaussian noise with standard deviation $\sigma_{v}=0.075 \mathrm{~m}$ was added to account for the measurement errors.

As starting prior distribution we chose: i) $p(\mathbf{z})$ with $w_{0}^{i}=$ $1 / N_{T}$ for $i=1, \ldots, N_{T}$, where $N_{T}$ is the number of integer candidate pairs $\left(z_{1}, z_{2}\right)$ (in this example $N_{T}=360$ ), according to the search space generation as illustrated in the Appendix ii) $p(x)$ with $\mu_{x, 0}=n_{0}$ and $\sigma_{x, 0}^{2}=(3 \mathrm{~m})^{2}$, being $n_{0}$ a randomly generated Gaussian perturbation with mean $\mu=x_{R}=10 \mathrm{~m}$ and standard deviation $\sigma_{x_{0}}=3 \mathrm{~m}$.

Once the data was generated, we started the sequential calculation of the posterior distribution. In Fig. 2, the evolution for different values of $k$ of the weights of the distribution $p\left(\mathbf{z} \mid \mathbf{y}_{1: k}\right)$ is shown on the left side whereas the posterior $p\left(\mathbf{x} \mid \mathbf{y}_{1: k}\right)$ is shown on the right side. On both sides of the Figure, the true value is highlighted. It can be clearly seen how the probability of the true ambiguity value grows as more measurements are used. Moreover, notice how the weights corresponding to other candidate pairs decrease. However, notice that the weight of the true value does not grow monotonically and the weights of the incorrect values do not decrease monotonically. Hence, in order to improve the reliability of the method, it becomes very important resorting to the decision threshold $\gamma$. Regarding the continuous part of the distribution, it can be seen how the shape of the distribution changes after new measurements are added. For the first measurements, the distribution is clearly multi-modal due to its Gaussian sum nature, where each component has a relatively high variance. As more measurements are included, the number of noticeable Gaussian components as well as their variance decrease.

\section{EXTENSION FOR TIME VARYING REAL PARAMETERS}

We now consider the case where the real parameters $\mathbf{x}$ vary with time. Assume that the parameters follow the linear dynamical model

$$
\mathbf{x}_{k+1}=\mathbf{F}_{k} \mathbf{x}_{k}+\mathbf{w}_{k}
$$

where $\mathbf{F}_{k}$ is the state transition matrix of $\mathbf{x}_{k}$ and $\mathbf{w}_{k}$ is the process noise that is assumed to be zero mean Gaussian with covariance matrix $\boldsymbol{\Sigma}_{\mathbf{w}, k}$.

Following the same derivation steps as in Section III, for a given prior distribution

$$
\begin{aligned}
p\left(\mathbf{x}_{k-1}, \mathbf{z} \mid \mathbf{y}_{1: k-1}\right) & \\
= & \sum_{i=1}^{N_{T}} \mathcal{N}\left(\mathbf{x}_{k-1} ; \boldsymbol{\mu}_{\mathbf{x}, k-1}^{i}, \boldsymbol{\Sigma}_{\mathbf{x}, k-1}\right) w_{k-1}^{i} \delta\left(\mathbf{z}-\mathbf{z}^{i}\right),
\end{aligned}
$$

the posterior distribution results

$$
p\left(\mathbf{x}_{k}, \mathbf{z} \mid \mathbf{y}_{1: k}\right)=\sum_{i=1}^{N_{T}} \mathcal{N}\left(\mathbf{x}_{k} ; \boldsymbol{\mu}_{\mathbf{x}, k}^{i}, \boldsymbol{\Sigma}_{\mathbf{x}, k}\right) w_{k}^{i} \delta\left(\mathbf{z}-\mathbf{z}^{i}\right)
$$

where

$$
\begin{aligned}
\boldsymbol{\mu}_{\mathbf{x}, k}^{i} & =\mathbf{F}_{k-1} \boldsymbol{\mu}_{\mathbf{x}, k-1}^{i}+\mathbf{K}_{k}\left(\mathbf{y}_{k}-\boldsymbol{\mu}_{\mathbf{y}, k}^{i}\right) \\
\boldsymbol{\Sigma}_{\mathbf{x}, k} & =\left(I-\mathbf{K}_{k} \mathbf{A}_{k}\right)\left(\mathbf{F}_{k-1} \boldsymbol{\Sigma}_{\mathbf{x}, k-1} \mathbf{F}_{k-1}^{T}+\boldsymbol{\Sigma}_{\mathbf{w}, k}\right)
\end{aligned}
$$

$$
\text { with } \begin{aligned}
\mathbf{K}_{k} & =\left(\mathbf{F}_{k-1} \boldsymbol{\Sigma}_{\mathbf{x}, k-1} \mathbf{F}_{k-1}^{T}+\boldsymbol{\Sigma}_{\mathbf{w}, k}\right) \mathbf{A}_{k}^{T} \boldsymbol{\Sigma}_{\mathbf{y}, k}^{-1} \\
\boldsymbol{\mu}_{\mathbf{y}, k}^{i} & =\mathbf{A}_{k} \mathbf{F}_{k-1} \boldsymbol{\mu}_{\mathbf{x}, k-1}^{i}+\mathbf{B}_{k} \mathbf{z}^{i} \\
\boldsymbol{\Sigma}_{\mathbf{y}, k} & =\mathbf{A}_{k} \mathbf{F}_{k-1} \boldsymbol{\Sigma}_{\mathbf{x}, k-1} \mathbf{F}_{k-1}^{T} \mathbf{A}_{k}^{T}+\boldsymbol{\Sigma}_{\mathbf{v}, k} \quad \text { and } \\
w_{k}^{i} & =\frac{w_{k-1}^{i} \mathcal{N}\left(\mathbf{y}_{k} ; \boldsymbol{\mu}_{\mathbf{y}, k}^{i}, \boldsymbol{\Sigma}_{\mathbf{y}, k}\right)}{\sum_{i=1}^{N_{T}} w_{k-1}^{i} \mathcal{N}\left(\mathbf{y}_{k} ; \boldsymbol{\mu}_{\mathbf{y}, k}^{i}, \boldsymbol{\Sigma}_{\mathbf{y}, k}\right)}
\end{aligned}
$$

Remark 1: Observe that (33)-(37) correspond to the single step update of a Kalman filter with dynamic model (30) and measurements (4). The $N_{T}$ posible values of $z$ shift the 1-step predictors, first term of the RHS of (36). Remark 2: Note that a new $\mathbf{K}_{k}$ matrix, that can be seen as a Kalman gain, appears in order to simplify $\boldsymbol{\mu}_{\mathbf{x}, k}^{i}$ and $\boldsymbol{\Sigma}_{\mathbf{x}, k}$ expressions. As in (20), it can also be seen that (32) is factored as $p\left(\mathbf{x}_{k}, \mathbf{z} \mid \mathbf{y}_{1: k}\right)=p\left(\mathbf{x}_{k} \mid \mathbf{z}, \mathbf{y}_{1: k}\right) p\left(\mathbf{z} \mid \mathbf{y}_{1: k}\right)$, with $p\left(\mathbf{x}_{k} \mid \mathbf{z}, \mathbf{y}_{1: k}\right)=$ $\mathcal{N}\left(\mathbf{x}_{k} ; \boldsymbol{\mu}_{\mathbf{x}, k}^{i}, \boldsymbol{\Sigma}_{\mathbf{x}, k}\right)$ and $p\left(\mathbf{z} \mid \mathbf{y}_{1: k}\right)=\sum_{i=1}^{N_{T}} w_{k}^{i} \delta\left(\mathbf{z}-\mathbf{z}^{i}\right)$. Once the posterior distribution is found, the estimators are obtained as in the static case through (26) and (27).

\section{AnAlysis of the TECHNiQUe}

\section{A. Comparison to Other Bayesian Estimation Techniques}

Considering (33), (34), (35), (36) and (37) in detail and dropping for a while the superindex $i$, it can be noted that they 

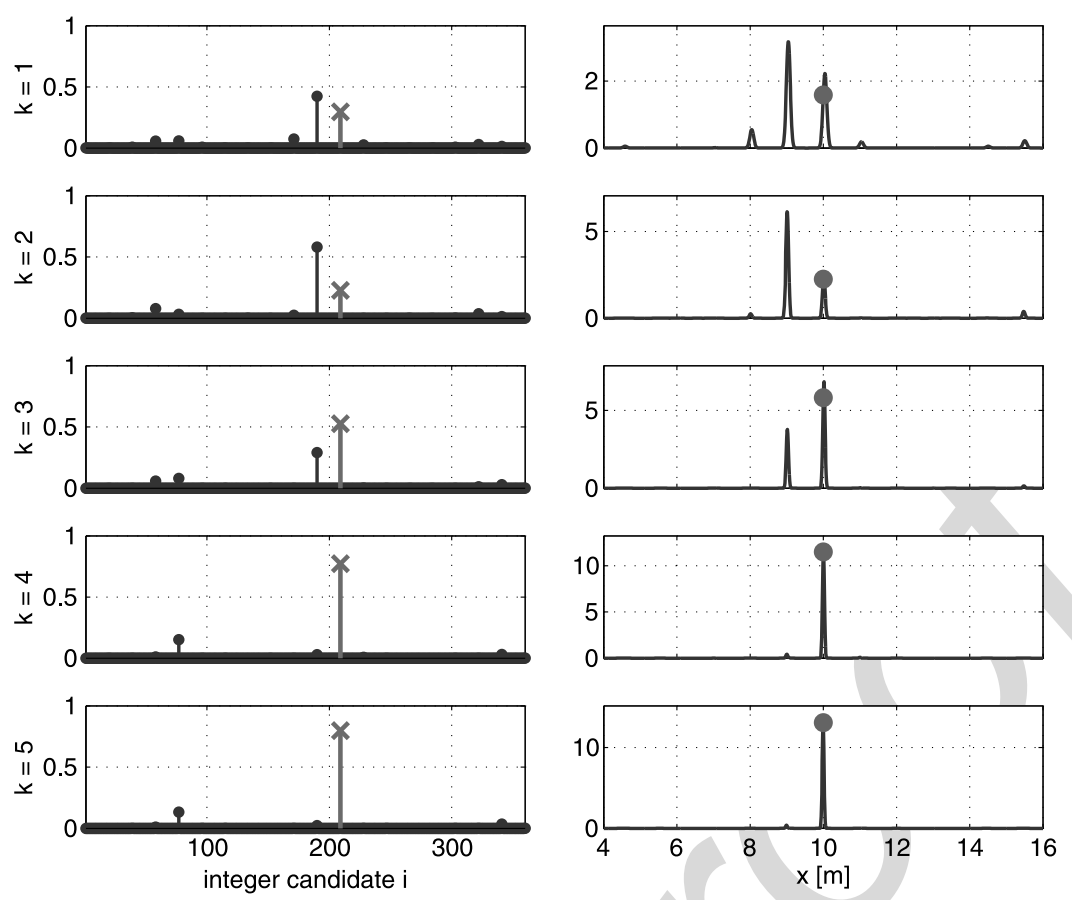

Fig. 2. Evolution for $k=1, \ldots, 5$ of $p\left(\mathbf{z} \mid \mathbf{y}_{1: k}\right)$ (left) and $p\left(\mathbf{x} \mid \mathbf{y}_{1: k}\right)$ (right). The correct value is highlighted.

are very similar to the Kalman filter equations for the estimation of the real parameters $\mathbf{x}$. The same happens with (21), (22), (23) and (24) for the time invariant real-parameter model, $\mathbf{x}_{k+1}=\mathbf{x}_{k}$. Since these equations are indexed by $i$, the developed technique can be interpreted as $N_{T}$ (the total number of possible integer vectors) Kalman filters for the real parameters running in parallel, each one corresponding to a different vector of integer values. However, the computational burden and the memory storage required by our method are far less than it would be needed for the implementation of this number of KFs. This is possible because the covariance matrices and Kalman gains computation are the same for all filters (see Fig. 3). Thus, only filtered and predicted values of each KF must be separately computed and stored. Moreover, the proposed technique can be thought of as an implementation of the Interacting Multiple Models filter [24] but instead of a weighted output, in this case the estimated value is directly one of the multiple filter outputs if the weight value of one of them is greater than a given threshold. Finally, the estimation method proposed in this paper could be considered among the Grid Based Methods in Bayesian Filtering [25]. Our technique for the real part of the state vector also resembles the "sum of Gaussians" approach used to approximate the prediction and measurement distributions in nonlinear filtering [26].

In the introduction we already mentioned that [13]-[17] also applied a Bayesian approach resembling ours, but notably different in optimality criterion, assumptions on the integer parameters, sequential implementation (save for [17]) and the possibility to adopt a dynamical model for the real parameters. It is worth noting that there exists a connection between our Bayesian and other non-Bayesian techniques. This holds true for Teunissen's theory of integer equivariant estimation and integer aperture estimation. In [9] it is shown for instance how the

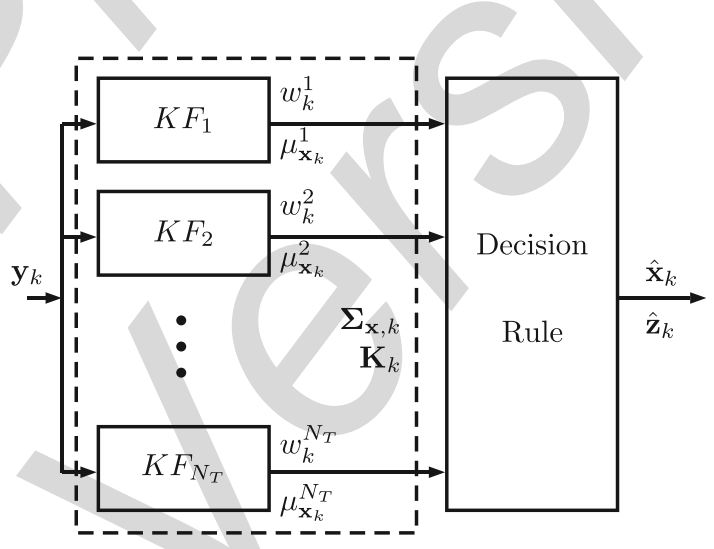

Fig. 3. Graphical interpretation of our method. It can be seen as $N_{T}$ Kalman filters $\left(K F_{i}\right)$ that share the same cov. matrix $\left(\boldsymbol{\Sigma}_{\mathbf{x}, k}\right)$ and Kalman gain $\left(\mathbf{K}_{k}\right)$ plus a decision stage that is function of $w_{k}^{i}$ with $i=1, \ldots, N_{T}$.

weights in his non-Bayesian BIE-estimator of the ambiguities can be directly linked to the Bayesian a posteriori probabilities. The link between the Bayesian approach and the optimal integer aperture estimation [11] is pointed out in [27]. There, the Bayesian a posteriori probability shows itself in the optimal aperture region. Also in [27] another interpretation is given connecting the Bayesian approach and the non-Bayesian penalized ambiguity estimator introduced in [28].

\section{B. Computational Requirements}

In order to assess the implementation feasibility, a quick computational requirement analysis is presented. As it was analyzed in the previous subsection, our technique is equivalent to $N_{T}$ KFs, but its computational requirements are far lower than those required for the same number of filters. For each time update, our method needs only one Kalman gain and only one covariance matrix computation. These are the most computationally 
expensive tasks of a KF because they require some matrix inversions. In addition, it requires $(i) N_{T}$ mean updates involving one matrix times vector multiplication and (ii) $N_{T}$ weight updates involving a scalar product, an exponential function evaluation of a quadratic form and an $N_{T}$ component vector normalization. Thus, the computational requirements of our method are similar to a $p$ state, $m$ measurement Kalman filter plus $N_{T}$ simple matrix multiplications and exponential function evaluations. This coarse analysis shows that the computational requirements of our method do not exceed by much those of a single KF and are easily achievable; especially so for embedded systems. Then, it seems suited for real-time systems.

\section{Application to GNSS High PRECision DifFERENTIAL POSITIONING}

The general model of (4) can be directly applied to the problem of GNSS high precision differential positioning. It consists in the sub-centimetric estimation of the difference of coordinates between two GNSS antennas with their respective receivers. Carrier phase differential measurements are typically used due to their high accuracy (in the order of the millimeters). However, these measurements are ambiguous in an integer number of carrier cycles and then, an ambiguity resolution technique should be used. Depending on the specific application and the quality of the receivers employed, unambiguous but noisier code measurements can also be used. Carrier-phase only or code and carrier-phase measurements can be used for more than one frequency in order to get better results. In order to cancel out errors common to both receivers the so-called double differences are frequently used [29], [30].

The code or carrier-phase double differences between satellites $j$ and $i$ at time $t_{k}$ are defined as

$$
\nabla \Delta M_{k}^{j i}=\left(M_{1, k}^{j}-M_{0, k}^{j}\right)-\left(M_{1, k}^{i}-M_{0, k}^{i}\right)
$$

where $M_{m, k}^{l}$ refers to either the carrier-phase $(\phi)$ or the code $(\rho)$ measurement corresponding to satellite $l$ in the receiver $m$ at time $t_{k}$. If the distance between receivers is short (less than 10 $\mathrm{km}$ ), carrier-phase and code double differences can be modeled as [29],

$$
\begin{aligned}
& \nabla \Delta \phi_{k}^{j i}=-\lambda \nabla \Delta N^{j i}-\left(\mathbf{s}_{k}^{j i}\right)^{T} \mathbf{r}_{k}+\eta_{k} \\
& \nabla \Delta \rho_{k}^{j i}=-\left(\mathbf{s}_{k}^{j i}\right)^{T} \mathbf{r}_{k}+\epsilon_{k}
\end{aligned}
$$

where $\lambda$ is the wavelength of the utilized GNSS signal (today's most frequent is the $L 1$ GPS signal with $\lambda \simeq 19 \mathrm{~cm}) ; \nabla \Delta N^{j i}$ is the integer wavelength ambiguity; $\mathbf{s}_{k}^{j i}$ is the difference between the line-of-sight (LOS) vector to satellite $j, \mathbf{s}^{j}$, and the corresponding to satellite $i, \mathbf{s}^{i} ; \mathbf{r}_{k}=\left[\Delta x_{k}, \Delta y_{k}, \Delta z_{k}\right]^{T}$. The latter is the baseline vector (i.e., the vector of the difference of coordinates between receivers); and $\eta_{k}$ and $\epsilon_{k}$ are the carrier-phase and the code measurement noise respectively, mainly involving thermal noise and multipath. If multipath is negligible, as it occurs in a good sky visibility condition and/or when appropriate antennas are used in a suitable environment, $\eta_{k}$ and $\epsilon_{k}$ can be modeled as Gaussian noise. Note that typically, the noise stan- dard deviation in code measurements is about 100 to 1000 times larger than in carrier-phase measurements.

If at time $t_{k}$ there are $n+1$ satellites in sight, and taking satellite $n+1$ as the reference satellite; $n$ independent double differences can be formed for each type of measurement and for each frequency. Their aggregation in vector form results in the measurement sample

$$
\begin{aligned}
\nabla \Delta \boldsymbol{\Phi}_{k} & =\mathbf{S}_{k}^{T} \mathbf{r}_{k}-\lambda \nabla \Delta \mathbf{N}+\boldsymbol{\eta}_{k}, \\
\nabla \Delta \Psi_{k} & =\mathbf{S}_{k}^{T} \mathbf{r}_{k}+\boldsymbol{\epsilon}_{k}, \\
\text { where } \quad \mathbf{S}_{k} & =\left[-\mathbf{s}_{k}^{1(n+1)}, \ldots,-\mathbf{s}_{k}^{n(n+1)}\right]
\end{aligned}
$$

is the line-of-sight difference matrix. Due to the satellite constellation configuration, $\mathbf{S}_{k}^{T}$ is generally full column rank (equal to 3). $\nabla \Delta \Phi_{k}=\left[\nabla \Delta \phi_{k}^{1(n+1)}, \ldots, \nabla \Delta \phi_{k}^{n n+1}\right]^{\mathrm{T}}$ is the carrier-phase double difference vector, $\nabla \Delta \Psi_{k}=$ $\left[\nabla \Delta \rho_{k}^{1(n+1)}, \ldots, \nabla \Delta \rho_{k}^{n n+1}\right]^{\mathrm{T}}$ is the code double difference vector and $\nabla \Delta \mathbf{N}$ is the integer wavelength ambiguity vector. The resulting measurement noise vectors $\boldsymbol{\eta}_{k}$ and $\boldsymbol{\epsilon}_{k}$ are zero mean Gaussian with covariance matrix $\mathbf{C}_{\phi}$ and $\mathbf{C}_{\rho}$ given by

$$
\begin{aligned}
& \mathbf{C}_{\phi}=\left(\mathbf{D}_{\phi}+\sigma_{\phi, n+1}^{2} \rrbracket_{n} \rrbracket_{n}^{T}\right) \\
& \mathbf{C}_{\rho}=\left(\mathbf{D}_{\rho}+\sigma_{\rho, n+1}^{2} \rrbracket_{n} \rrbracket_{n}^{T}\right)
\end{aligned}
$$

where $\mathbf{D}_{\phi}=\operatorname{diag}\left[\sigma_{\phi, 1}^{2} \ldots \sigma_{\phi, n}^{2}\right]$, with $\sigma_{\phi, i}^{2}$ being the variance of the $i$-th carrier-phase measurement, $\mathbf{D}_{\rho}=\operatorname{diag}\left[\sigma_{\rho, 1}^{2} \ldots \sigma_{\rho, n}^{2}\right]$, with $\sigma_{\rho, i}^{2}$ being the variance of the $i$-th code measurement, $I_{n}$ is the $n \times n$ identity matrix and $\rrbracket_{n}$ is $n$-dimensional vector with all ones, $[1, \ldots, 1]^{T}$. Equations (44) and (45) assume that each carrier-phase measurement noise and each code measurement noise are independent among measurements. The structure of the matrix reflects the correlation arising from the double differences [31].

As it was previously mentioned, in the context of high precision GNSS differential positioning there arise different models that correspond to different types of measurements. These, in turn depend on the quality of the receiver employed. The simplest model uses only Single-Frequency Carrier-Phase measurements and is denoted SFCP. This model is useful for very short baselines, typically used for attitude estimation, where code measurements provide useless information due to its high noise level. In this case, it is easily seen from (42) and comparing it with (4) that our technique fits to this problem taking $\mathbf{y}_{k}=\nabla \Delta \boldsymbol{\Phi}_{k}, \mathbf{x}_{k}=\mathbf{r}_{k}, \mathbf{z}=\nabla \Delta \mathbf{N}, \mathbf{A}_{k}=\mathbf{S}_{k}^{T}$, $\mathbf{B}_{k}=-\lambda I_{n}$ and $\boldsymbol{\Sigma}_{\mathbf{v}, k}=\mathbf{C}_{\phi}$. The number of real parameters to be estimated is $p=3$, whereas the integer parameters are $q=n$. Usually, this model is also used for short baselines (up to a few kilometers). However, for this kind of baselines, the Single-Frequency Carrier-Phase and Code (SFCPC) model can also be used in order to take advantage of code information. In this case $\mathbf{y}_{k}=\left[\nabla \Delta \boldsymbol{\Psi}_{k}^{T}, \nabla \Delta \boldsymbol{\Phi}_{k}^{T}\right]^{T}, \mathbf{x}_{k}=\mathbf{r}_{k}, \mathbf{z}=\nabla \Delta \mathbf{N}$, $\mathbf{A}_{k}=\left[\mathbf{S}_{k}, \mathbf{S}_{k}\right]^{T}, \mathbf{B}_{k}=\left[\mathbf{0}_{n},-\lambda I_{n}\right]^{T}$ and

$$
\boldsymbol{\Sigma}_{\mathbf{v}, k}=\left[\begin{array}{ll}
\mathbf{C}_{\rho} & \mathbf{0}_{n} \\
\mathbf{0}_{n} & \mathbf{C}_{\phi}
\end{array}\right]
$$


with $\mathbf{0}_{n}$ a $n \times n$ matrix of zeros. The number of real parameters to be estimated is the same as in the previous case, i.e., $p=3$ and $q=n$.

Finally, for long baseline estimation, spanning from a few to thousands of kilometers and requiring mitigation of iono-/tropo-spheric effects, high-end dual- (or multi-) frequency receivers are required. In this context, a simplified Dual-Frequency Carrier-Phase and Code (DFCPC) model results in $\mathbf{y}_{k}=\left[\nabla \Delta \boldsymbol{\Psi}_{L 1, k}^{T}, \nabla \Delta \boldsymbol{\Phi}_{L 1, k}^{T}, \nabla \Delta \boldsymbol{\Psi}_{L 2, k}^{T}, \nabla \Delta \boldsymbol{\Phi}_{L 2, k}^{T}\right]^{T}$, $\mathbf{x}_{k}=\mathbf{r}_{k}, \mathbf{z}=\left[\nabla \Delta \mathbf{N}_{L 1}^{T}, \nabla \Delta \mathbf{N}_{L 2}^{T}\right]^{T}, \mathbf{A}_{k}=\left[\mathbf{S}_{k}, \mathbf{S}_{k}, \mathbf{S}_{k}, \mathbf{S}_{k}\right]^{T}$,

$$
\begin{aligned}
\mathbf{B}_{k}= & {\left[\begin{array}{ccc}
\mathbf{0}_{n} & \mathbf{0}_{n} \\
-\lambda_{L 1} I_{n} & \mathbf{0}_{n} \\
\mathbf{0}_{n} & \mathbf{0}_{n} \\
\mathbf{0}_{n} & -\lambda_{L 2} I_{n}
\end{array}\right] \text { and } } \\
\boldsymbol{\Sigma}_{\mathbf{v}, k}= & {\left[\begin{array}{cccc}
\mathbf{C}_{\rho, L 1} & \mathbf{0}_{n} & \mathbf{0}_{n} & \mathbf{0}_{n} \\
\mathbf{0}_{n} & \mathbf{C}_{\phi, L 1} & \mathbf{0}_{n} & \mathbf{0}_{n} \\
\mathbf{0}_{n} & \mathbf{0}_{n} & \mathbf{C}_{\rho, L 2} & \mathbf{0}_{n} \\
\mathbf{0}_{n} & \mathbf{0}_{n} & \mathbf{0}_{n} & \mathbf{C}_{\phi, L 2}
\end{array}\right] . }
\end{aligned}
$$

In this simplified model, the number of real parameters to be estimated is $p=3$ since only the baseline Cartesian components are to be estimated. For long baselines of more than 10 $\mathrm{km}$, more real parameters are typically required, such as ionosphere and troposphere delays, receiver and satellite clocks and instrumental delays. In these cases, $\mathbf{A}_{k}$ should be modified accordingly. The number of the integer parameters is $q=2 n$.

Note that depending on the kind of problem with regards to the baseline (i.e., static or kinematic) one of the approaches of Section III or Section IV must be used.

In the sequel, when our method is used in the context of GNSS Ambiguity Resolution we will refer to it as BART for Bayesian Ambiguity Resolution Technique.

\section{SiMULATION RESULTS}

In this section, we present some simulations for the GNSS high precision positioning problem described in the previous section in three different cases: i) single-frequency (GPS L1) carrier-phase measurements only (SFCP), ii) single-frequency (GPS L1) carrier-phase and code measurements (SFCPC) and iii) dual-frequency (GPS L1 and L2) carrier-phase and code (DFCPC).

For the simulations, measurements were synthetically generated by means of our own simulation routines using real broadcast ephemeris data of the GPS constellation. A $5 \mathrm{~km}$ static baseline was considered. The simulated data assumed ideal conditions (i.e., no atmospheric or other perturbation effects were considered). The correlation in the double differences was taken into account for the noise generation. The same noise variance was used for all satellites but different for each kind of measurements (i.e., carrier-phase and code), so the noise elevation dependency was neglected. The simulation parameters are summarized in Table I, where ECEF stands for the Earth Centered Earth Fixed coordinate frame used as reference in the GPS.

In addition to BART results, outcomes obtained via the LAMBDA method using the same synthetic data are also shown for comparison purposes. The LAMBDA method is one of the most famous and successful ambiguity resolution
TABLE I

GNSS DIFFERENTIAL POSITIONING SIMULATION PARAMETERS

\begin{tabular}{c|c} 
Parameter & Value \\
\hline \hline Reference Position $(\mathrm{ECEF})[\mathrm{km}]$ & {$[2779.3,-4437.9,-3629.4]$} \\
\hline Baseline Cartesian comp. $(\mathrm{ECEF})[\mathrm{m}]$ & {$[3191.4,-1047.9,3703.7]$} \\
\hline Carrier Phase Noise st. dev. $\left(\sigma_{\phi}\right)[\mathrm{m}]$ & 0.002 \\
\hline Code Noise st. dev. $\left(\sigma_{\rho}\right)[\mathrm{m}]$ & 1 \\
\hline
\end{tabular}

methods in the GNSS literature [32], [33]. A detailed description of the algorithm is given in [34]. The results presented in this paper were obtained using the LAMBDA software package ver. 3.0 [35].

In order to analyze performance, several Monte-Carlo (MC) runs with different simulation scenarios were done for each of the three cases considered. Each scenario is defined by the number of measurements used and the chosen threshold level. We varied the number of measurements from 4 (5 satellites in sight) to 7 ( 8 satellites in sight) and we considered three different values for the threshold $\gamma, 0.75,0.9$ and 0.99 . Each scenario consists of $K=200$ samples (or measurement epochs) taken at a sample rate of $1 \mathrm{~Hz}$, and it is repeated 10000 times.

Three performance measures are considered: $(i)$ the success rate (SR), which accounts for the number of correct ambiguity estimations; ( $i i)$ the error rate (ER), for the number of incorrect ambiguity estimations; and (iii) the mean time to make a decision (MTMD), which indicates the number of measurement epochs (that is the efficiency of the method) required until a valid estimate is given.

\section{A. BART Simulation Results}

As starting prior distribution for the BART method we chose: (i) $p(\mathbf{z})$ with $w_{0}^{i}=1 / N_{T}$ for $i=1, \ldots, N_{T}$, where $N_{T}$ is the number of elements of the ambiguity candidate set that is constructed as explained in Appendix A with $\alpha=0.01$, the covariance matrix for $z$ is the same as that used with the LAMBDA method in the following section; (ii) $p(\mathbf{x})$ with $\boldsymbol{\mu}_{\mathbf{x}, 0}=\mathbf{n}_{0}$ and $\boldsymbol{\Sigma}_{\mathbf{x}, 0}=\sigma_{x_{0}}^{2} I_{3}$ with $\sigma_{x_{0}}=5 \mathrm{~m}, \mathbf{n}_{0}$ being a randomly generated perturbation whose mean is the true value of $\mathbf{x}$ plus a realization of a zero mean Gaussian random variable with standard deviation $\sigma_{x_{0}}$ in each component.

Once the data were generated and the ambiguity candidates set or search space (SS) was constructed, we proceeded with the sequential calculation of the posterior distribution. For each measurement epoch, the updated value of $p(\mathbf{z})$ is evaluated, if one of the candidates achieves a weight $w^{i}$ larger than the threshold $\gamma$ the estimation process is finished and the estimated integer and real values are returned.

The results of these simulations are summarized in Table II, each row corresponds to a different scenario. The first column indicates the case; the second, the number of double difference (DD) measurements used; the third, the threshold level; the fourth, the mean number of candidates (i.e., $N_{T}$ ) of the generated search space for each MC run and its standard deviation (between brackets); success and error rates (in percent units), as well as the MTMD (in seconds) are displayed in the 5th, 6th and $7 \mathrm{~h}$ columns respectively. Results from the DFCPC case were 
TABLE II

BART PERFORMANCE

\begin{tabular}{|c|c|c|c|c|c|c|}
\hline Case & $\begin{array}{l}\# \text { of } \\
\mathrm{DD}\end{array}$ & $\gamma$ & $\begin{array}{l}\text { SS \# of cand. } \\
\text { mean / (s.d.) }\end{array}$ & $\begin{array}{l}\mathrm{SR} \\
{[\%]}\end{array}$ & $\begin{array}{l}\text { ER } \\
{[\%]}\end{array}$ & $\begin{array}{l}\text { MTMD } \\
\text { [sec.] }\end{array}$ \\
\hline \multirow{12}{*}{ SFCP } & 4 & 0.75 & $2966.7 /(12.4)$ & 80.7 & 19.3 & 28.7 \\
\hline & 4 & 0.90 & $2966.5 /(12.3)$ & 92.6 & 7.4 & 32.4 \\
\hline & 4 & 0.99 & $2966.7 /(12.4)$ & 99.5 & 0.5 & 37.7 \\
\hline & 5 & 0.75 & $179.2 /(13.5)$ & 79.2 & 20.8 & 17.6 \\
\hline & 5 & 0.90 & $178.8 /(13.4)$ & 92.9 & 7.1 & 22.2 \\
\hline & 5 & 0.99 & $178.9 /(13.5)$ & 99.2 & 0.8 & 29.6 \\
\hline & 6 & 0.75 & $11.4 /(2.6)$ & 84.2 & 15.9 & 3.6 \\
\hline & 6 & 0.90 & $11.4 /(2.6)$ & 94.0 & 6.0 & 5.1 \\
\hline & 6 & 0.99 & $11.4 /(2.6)$ & 99.5 & 0.5 & 8.3 \\
\hline & 7 & 0.75 & $1.8 /(0.6)$ & 92.1 & 7.9 & 1.3 \\
\hline & 7 & 0.90 & $1.8 /(0.6)$ & 96.5 & 3.5 & 1.6 \\
\hline & 7 & 0.99 & $1.8 /(0.6)$ & 99.7 & 0.3 & 2.3 \\
\hline \multirow{12}{*}{ SFCPC } & 4 & 0.75 & $2966.8 /(12.4)$ & 82.8 & 17.2 & 15.1 \\
\hline & 4 & 0.90 & $2966.6 /(12.6)$ & 93.7 & 6.3 & 18.2 \\
\hline & 4 & 0.99 & $2966.4 /(12.6)$ & 99.4 & 0.6 & 23.4 \\
\hline & 5 & 0.75 & $179.0 /(13.4)$ & 82.7 & 17.3 & 8.7 \\
\hline & 5 & 0.90 & $179.0 /(13.4)$ & 93.7 & 6.3 & 12.0 \\
\hline & 5 & 0.99 & $179.0 /(13.5)$ & 99.3 & 0.7 & 17.7 \\
\hline & 6 & 0.75 & $11.4 /(2.6)$ & 85.6 & 14.4 & 2.7 \\
\hline & 6 & 0.90 & $11.4 /(2.6)$ & 95.2 & 4.8 & 4.2 \\
\hline & 6 & 0.99 & $11.4 /(2.6)$ & 99.5 & 0.5 & 6.9 \\
\hline & 7 & 0.75 & $1.9 /(0.6)$ & 97.4 & 2.6 & 1.1 \\
\hline & 7 & 0.90 & $1.8 /(0.6)$ & 99.1 & 0.9 & 1.2 \\
\hline & 7 & 0.99 & $1.8 /(0.6)$ & 99.9 & 0.1 & 1.4 \\
\hline
\end{tabular}

omitted because in all scenarios an instantaneous correct solution was achieved so that its SR is always $100 \%$ and its MTMD is always $1 \mathrm{sec}$. (In the GPS/GNSS literature, instantaneous ambiguity resolution customarily means that the ambiguities were estimated using only one measurement sample).

The results displayed in Table II clearly show the virtues of our method, as well as the impact of the selection of the threshold level $\gamma$. Anyway, recall that it is here applied to a simplified observation model that does not incorporate atmospheric and other effects. As expected from the discussion in Section III-C, in all of the presented scenarios the value of $\gamma$ resulted to be an empirical lower bound for the SR (i.e., for $\gamma=0.90, S R \geq 90 \%$ ). This makes the BART method not only an interesting estimation method but also a robust one because it intrinsically includes a validation stage that provides a given minimum SR through the selection of a proper $\gamma$. As it was previously mentioned, this is a nice property to have in any integer estimation algorithm. In addition, it can be noticed how the MTMD and the SS size vary depending on the value of $\gamma$ and on the scenario. As it is easily anticipated, the higher the value of $\gamma$ the longer the MTMD. On the other hand, it can be observed how the SS becomes larger when the underlying model is weak as in the case when there are few satellites in view and few types of measurements.

We remark that even in a very demanding condition as is the case with only 4 measurements of only GPS L1 carrier-phase, the method has an acceptable behavior regarding their MTMD and when there are 7 measurements (or 8 satellites present), a commonly met condition in practice these days, the SR and the MTMD are excellent allowing an almost always instantaneous solution. Moreover, when additional types of measurements are included, this behavior improves noticeably.

\section{B. LAMBDA Simulation Results}

The same synthetic data set was used to evaluate the performance of the LAMBDA method. Since it is a non-Bayesian method, BART simulation uses a-priori information that cannot be incorporated to LAMBDA. In addition, LAMBDA by itself doesn't provide a validation method that allows the user to determine if the solution obtained is good enough to be used or it should be discarded. There exist several validation methods discussed in the literature such as the Ratio Test, the Projection Test, the Difference Test, etc. We used the Ratio Test (RT) of [12] because it provides a fixed failure probability $\left(P_{f}\right)$ approach and its implementation for $P_{f}=0.01$ and $P_{f}=0.001$ is included in the LAMBDA software package used. For these simulations, we used both values of $P_{f}$.

The simulation process used is as follows: for a given start measurement epoch $(i)$ a float solution of the ambiguities (i.e., a solution that doesn't take into account the integer nature) and its covariance matrix is found through a Weighted Least Squares (WLS) method with the available measurements; (ii) the LAMBDA method is run with the estimates of (i); (iii) the Ratio Test is applied to the LAMBDA solution. If the RT passes, the process finishes. The number of measurement samples required is stored and the result is compared to the true one. If the RT does not pass, a new measurement sample is incorporated and the process starts again repeating steps (i), (ii) and (iii). It has to be noted that for the case SFCP, at least two measurement epochs are required in order to get the float solution due to a dimensionality problem (i.e., with only one measurement epoch we have more unknowns than measurements).

Table III shows the same information that in BART's Table II with the exception of $P_{f}$ instead of $\gamma .10,000 \mathrm{MC}$ runs were also used per row. Similarly to BART's simulations, the DFCPC case was omitted because in all scenarios an instantaneous correct solution was achieved. It can be noted that in all the considered scenarios, the ER is greater than the expected one based on the chosen $P_{f}$. This may be due to the constant selection method used in the RT as a function of the $P_{f}$. As it is obtained through numerical simulations using some values for the code and carrier-phase noise levels and some satellite configuration, it is likely that the empirical $P_{f}$ (i.e., ER) obtained in our simulations doesn't match the selected one. Note that for scenarios with more satellites, this mismatch decreases.

\section{Comparison of BART and LAMBDA Simulation Results}

Comparing the results of Table II and Table III it can be noticed that when the amount and variety of available measurements is large, both methods performs equally well (e.g., the DFCPC case). However, in situations with fewer types of measurements (e.g., SFCP and SFCFC) the BART method performs better than LAMBDA in terms of MTMD for the same expected SR. See for example the scenario of 7 DD in the SFCP case with 
TABLE III

LAMBDA (With RATIO TEST) PERFormance

\begin{tabular}{|c|c|c|c|c|c|}
\hline Case & \# of DD & $P_{f}$ & SR [\%] & ER [\%] & MTMD [sec.] \\
\hline \hline \multirow{5}{*}{ SFCP } & 4 & 0.01 & 95.97 & 4.03 & 35.6 \\
\cline { 2 - 6 } & 4 & 0.001 & 99.81 & 0.19 & 41.9 \\
\cline { 2 - 6 } & 5 & 0.01 & 94.16 & 5.84 & 24.9 \\
\cline { 2 - 6 } & 5 & 0.001 & 99.22 & 0.78 & 31.8 \\
\cline { 2 - 6 } & 6 & 0.01 & 97.28 & 2.72 & 8.7 \\
\cline { 2 - 6 } & 6 & 0.001 & 99.67 & 0.33 & 10.8 \\
\cline { 2 - 6 } & 7 & 0.01 & 98.96 & 1.04 & 5.2 \\
\cline { 2 - 6 } & 7 & 0.001 & 99.87 & 0.13 & 6.1 \\
\hline \multirow{5}{*}{ SFCPC } & 4 & 0.01 & 95.50 & 4.50 & 19.7 \\
\cline { 2 - 6 } & 4 & 0.001 & 99.68 & 0.32 & 25.8 \\
\cline { 2 - 6 } & 5 & 0.01 & 94.58 & 5.42 & 13.9 \\
\cline { 2 - 6 } & 6 & 0.001 & 99.37 & 0.63 & 19.9 \\
\cline { 2 - 6 } & 6 & 0.01 & 95.90 & 4.10 & 5.0 \\
\cline { 2 - 6 } & 7 & 0.001 & 99.32 & 0.68 & 8.0 \\
\cline { 2 - 6 } & 7 & 0.001 & 98.92 & 1.08 & 1.3 \\
\hline
\end{tabular}

$\gamma=0.99$, where the BART method achieves an excellent ambiguity resolution, SR is $99.7 \%$ and MTMD is $2.3 \mathrm{sec}$.; whereas for LAMBDA with RT with $P_{f}=0.01, \mathrm{SR}$ is $99 \%$ and MTMD is 5.2 sec., and with $P_{f}=0.001$, SR is $99.9 \%$ and MTMD is 6.1 sec.. In both cases requiring two to three times more measurement samples for almost the same SR. The difference observed in other scenarios, although it is noticeable, is not as significant as in the aforementioned case but always favoring the BART method. Besides this better behavior in the most demanding scenarios, a remarkable advantage of the BART method is that, if the underlying model is appropriate, a given performance can be achieved through the appropriate selection of the threshold level $\gamma$.

\section{CONCLUSIONS}

A novel Bayesian technique to jointly estimate integer and real parameters in a linear model of measurements has been proposed. It is assumed that the integer parameters belong to a finite set and that the real ones come from a realization of a Gaussian random vector. The method sequentially determines the posterior distribution of the parameters to be estimated as new measurements are incorporated. This posterior distribution is a mixed one, with a Gaussian continuous part and a discrete part that accounts for the probability of each of the elements of the finite set. A MAP estimator with the addition of a confidence threshold is used for the integer parameters and a MMSE estimator for the real parameters.

Our technique starts with an initial estimate of the real parameters, a covariance matrix that carries information about its accuracy, and a set of potential integer vector candidates with the same probability. Each time a new measurement is available, the posterior distribution is updated and the integer vector with the maximum probability is tested. Measurements are incorporated in this way up to the moment when this probability becomes greater than the threshold. Then, this last maximum probability vector is declared as the integer estimate and the corresponding normal mean is taken as the real estimate. It is interesting to note that the developed technique is equivalent to $N_{T}$ (the total number of possible integer vectors) Kalman filters estimating only the real parameters running in parallel, each one corresponding to a different vector of integer values. However, the computational burden and the memory storage required by our method are considerably lower because only the mean of each KF and its corresponding weight must separately be computed and stored.

The method was applied to differential positioning using GNSS signals and was named BART. At present, only simulated observations were considered in the frame of a simplified observation model, that do not consider atmospheric and other effects impacting the real GNSS observations. Several Monte-Carlo simulations were done for the GPS system considering different satellite visibility scenarios and different combinations of measurements (i.e., code and carrier-phase) as well as frequencies ( $\mathrm{L} 1$ and $\mathrm{L} 2$ ). With the same simulated data, the results obtained were compared to those obtained with the widely used LAMBDA method with the addition of the Ratio Test as a validation stage. The simulation comparisons favored BART algorithm. This is particularly noticeable in demanding scenarios (e.g., those with only carrier-phase measurements of a single frequency), and when performance is measured by success rate, error rate, and mean time to make a decision. Both LAMBDA and BART perform similarly when there are several types of measurements (i.e., carrier-phase and code of more than one frequency) and the number of measurements is high. In addition, the simulations showed the effect of the threshold $\gamma$. As expected from the method derivation, it was found that $\gamma$ acts as a lower bound for the SR allowing to control the reliability of the estimation process through the selection of this value. This selection impacts in the MTMD, values near to 1 increase the SR but at the expense of a higher MTMD. On the other hand, lower values of $\gamma$ decrease the MTMD at the expense of a higher ER. Thus, our method not only provides an estimation stage but also a validation stage with a guaranteed SR. This very interesting property arises from the probabilistic model employed and was observed empirically from the simulation results. However, a more detailed theoretical analysis -that exceeds the scope of this work- should be carried out to confirm this result in a general sense.

Future work will involve an evaluation of BART with real data in different scenarios, with static and kinematic baselines. Also, a performance evaluation is to be done in other scenarios such as the estimation of long baselines with dual (or triple) frequency measurements. A current concern in this kind of applications is the emergence of biases that challenge the ambiguity resolution. It is foreseen that through a good modeling of this effect, BART could be successfully used under such challenges.

\section{APPENDIX \\ SEARCH SpaCe Generation}

A key point of our technique is to properly generate the set of all the integer potential candidates or search space. To do that we need an initial estimate of the integer parameters $\hat{\mathbf{z}}_{0}$, that could be real (i.e., in the GNSS/GPS literature known as the ambiguity float solution) and their covariance matrix $\boldsymbol{\Sigma}_{\hat{\mathbf{z}}_{0}}$ based on the prior information available. The basis of the procedure is 
that the quadratic form of the $\mathbf{z}$ residuals can be considered as a $\chi^{2}$ distribution with $q$ degrees of freedom. Thus, an hyper-ellipsoid enclosing the true ambiguity candidate with probability $(1-\alpha)$ can be constructed as

$$
\left(\hat{\mathbf{z}}_{0}-\mathbf{z}\right)^{T} \boldsymbol{\Sigma}_{\hat{\mathbf{z}}_{0}}^{-1}\left(\hat{\mathbf{z}}_{0}-\mathbf{z}\right) \leq \chi_{q}^{2}(1-\alpha),
$$

where $\chi_{q}^{2}(1-\alpha)$ is the $1-\alpha$ percentile of the chi-square with $q$ degrees of freedom cumulative distribution function (cdf). In order to find all the potential candidates in an efficient way, a triangular $L^{T} D L$ decomposition of $\boldsymbol{\Sigma}_{\hat{\mathbf{z}}_{0}}$ can be used. The procedure is somewhat similar to the DECODE algorithm of [1] or the search section of the LAMBDA [34] method but without any function evaluation so that it returns all integer candidates inside the ellipsoid. When $q$ increases, the shape of the ellipsoid in (46) becomes elongated and thus difficult to go over. A convenient solution to this problem is to decorrelate $\mathbf{z}$ using a transformation to make $\boldsymbol{\Sigma}_{\hat{\mathbf{z}}_{0}}$ as close to diagonal as possible while preserving the integer nature of the transformed integer parameters. In this way the shape of the search space is close to spherical making it easy to go over. The transformation $T$ should be an admissible transformation [32]: $\mathbf{u}=\mathbf{T z}$. where $\mathbf{u}=\left[u_{1}, \ldots, u_{q}\right]$ is the decorrelated integer parameter vector. To be an admissible transformation $\mathbf{T}$ must satisfy that both $\mathbf{T}$ and its inverse $\mathbf{T}^{-1}$ have integer entries. After the transformation, $\hat{\mathbf{z}}_{0}$ becomes $\hat{\mathbf{u}}_{0}=\mathbf{T} \hat{\mathbf{z}}_{0}$ and the new covariance matrix results $\boldsymbol{\Sigma}_{\hat{\mathbf{u}}_{0}}=\mathbf{T} \boldsymbol{\Sigma}_{\hat{\mathbf{z}}_{0}} \mathbf{T}^{T}$. Using the previously mentioned triangular decomposition $\boldsymbol{\Sigma}_{\hat{\mathbf{u}}_{0}}=\mathbf{L}^{T} \mathbf{D L}$, the transformed version of (46) can be expressed as

$$
\sum_{j=1}^{q} \frac{\left(\tilde{u}_{0, j}-u_{j}\right)^{2}}{d_{j}} \leq \chi_{q}^{2}(1-\alpha)
$$

where

$$
\tilde{u}_{0, i}=\left\{\begin{array}{cc}
\hat{u}_{0, i}-\sum_{j=i+1}^{q}\left(\tilde{u}_{0, j}-u_{j}\right) l_{j, i} & 1 \leq i \leq q-1 \\
\hat{u}_{0, q} & i=q
\end{array}\right.
$$

being $d_{i}$ the $i$-th element of the diagonal of $\mathbf{D}$ and $l_{j, i}$ the $j$-th row, $i$-th column entry of $\mathbf{L}$. From (48) $q$ intervals which are used for the search space generation can be constructed as

$$
\begin{gathered}
\tilde{u}_{0, q}-d_{q} \chi \leq u_{q} \leq \tilde{u}_{0, q}+d_{q} \chi \\
\vdots \\
\tilde{u}_{0, i}-\Delta u_{i} \leq u_{i} \leq \tilde{u}_{0, i}+\Delta u_{i} \\
\vdots \\
\tilde{u}_{0,1}-\Delta u_{1} \leq u_{1} \leq \tilde{u}_{0,1}+\Delta u_{1}
\end{gathered}
$$

where

$$
\Delta u_{i}=\sqrt{d_{i}\left(\chi^{2}-\sum_{j=i+1}^{q} \frac{\left(u_{j}-\tilde{u}_{0, j}\right)^{2}}{d_{j}}\right)} .
$$

Notice that $\chi$ was used instead of $\chi_{q}(1-\alpha)$ to improve the legibility of the equations.

Once the search space is generated for the transformed integer parameter vector $\mathbf{u}$, each component $\mathbf{u}^{i}$ is transformed back to the $\mathbf{z}$ variable through the inverse transformation (i.e., $\mathbf{z}^{i}=\mathbf{T}^{-1} \mathbf{u}^{i}$ ). This is the search space needed. It is important to note that the Search Space remains the same provided that there is no cycle slips or satellite changes.

From the previous discussion it can be seen that accurate values of $\hat{\mathbf{z}}_{0}$ and $\boldsymbol{\Sigma}_{\hat{\mathbf{z}}_{0}}$ are needed to generate the search space. Here we will discuss how to do it when we have some prior information about $\mathbf{x}$. Assume we have $\hat{\mathbf{x}}_{0}$, an estimate of $\mathbf{x}$ with covariance matrix $\boldsymbol{\Sigma}_{\hat{\mathbf{x}}, 0}$. From (4) we can get a real estimate $\hat{\mathbf{z}}_{0}$ of $\mathbf{z}$ from some measurement $\mathbf{y}_{k}$ as

$$
\hat{\mathbf{z}}_{0}=\left(\mathbf{B}_{k}^{T} \mathbf{B}_{k}\right)^{-1} \mathbf{B}_{k}^{T}\left(\mathbf{y}_{k}-\mathbf{A}_{k} \hat{\mathbf{x}}_{0}\right)
$$

with covariance matrix

$$
\boldsymbol{\Sigma}_{\hat{\mathbf{z}}_{0}}=\left(\mathbf{B}_{k}^{T} \mathbf{B}_{k}\right)^{-1} \mathbf{B}_{k}^{T}\left(\boldsymbol{\Sigma}_{v}+\mathbf{A}_{k} \boldsymbol{\Sigma}_{\hat{\mathbf{x}}, 0} \mathbf{A}_{k}^{T}\right) \mathbf{B}_{k}\left(\mathbf{B}_{k}^{T} \mathbf{B}_{k}\right)^{-1}
$$

That is the information that we were looking for.

\section{ACKNOWLEDGMENT}

The first author would like to thank the Argentine Government BEC.AR Program and the Argentine Fulbright Commission for the short term fellowship to visit the University of Colorado Boulder, and Prof. Penny Axelrad for her valuable comments on the manuscript and her commitment towards making this visit possible.

\section{REFERENCES}

[1] E. Agrell, T. Eriksson, A. Vardy, and K. Zeger, "Closest point search in lattices," IEEE Trans. Inf. Theory, vol. 48, no. 8, pp. 2201-2214, 2002.

[2] A. Hassibi and S. Boyd, "Integer parameter estimation in linear models with applications to GPS," IEEE Trans. Signal Process., vol. 46, no. 11, pp. 2938-2952, Nov. 1998.

[3] A. Korkine and G. Zolotareff, "Sur les formes quadratiques," Math. Ann., vol. 6, no. 3, pp. 366-389, 1873.

[4] A. Lenstra, H. Lenstra, and L. Lovasz, "Factoring polynomials with rational coefficients," Math. Ann., vol. 261, no. 4, pp. 515-534, 1982.

[5] Y. Al-Haifi, S. Corbrtt, and P. Cross, "Performance evaluation of GPS single-epoch en-the-fly ambiguity resolution," J. Inst. Navigation, vol. 44, no. 4, pp. 479-487, 1998.

[6] B. Remondi, "Pseudo kinematic GPS results using the ambiguity function method," J. Inst. Navigation, vol. 38, no. 1, pp. 17-36, 1991.

[7] R. Hatch, "Instantaneous ambiguity resolution," in Proc. IAG Symp. No 107, Kinematic Systems in Geodesy Surveying, and Remote Sensing, September 1990, pp. 299-308.

[8] H. Lenstra, "Integer programming with a fixed number of variables," Math. Oper. Res., 1981.

[9] P. Teunissen, "Theory of integer equivariant estimation with application to GNSS," J. Geodesy, vol. 77, no. 7-8, pp. 402-410, 2003.

[10] S. Verhagen and P. Teunissen, "Integer aperture GNSS ambiguity resolution,” Artificial Satellites, vol. 38, pp. 79-88, 2003.

[11] S. Verhagen and P. Teunissen, "GNSS ambiguity resolution with optimally controlled failure-rate," Artificial Satellites, vol. 40, no. 4, pp. 219-227, 2005

[12] S. Verhagen and P. Teunissen, "The ratio test for future GNSS ambiguity resolution," GPS Solutions vol. 17, no. 4, pp. 535-548, 2013 [Online]. Available: http://dx.doi.org/10.1007/s10291-012-0299-z

[13] B. Betti, M. Crespi, and F. Sansò, "A geometric illustration of ambiguity resolution in GPS theory and a Bayesian approach," Manuscripta Geodaetica, vol. 18, pp. 317-330, 1993. 
[14] J. Zhu, X. Ding, and Y. Chen, "Maximum-likelihood ambiguity resolution based on Bayesian principle," J. Geodesy, vol. 75, pp. 175-187, 2001.

[15] M. de Lacy, F. Sansò, G. Rodriguez-Caderot, and A. J. Gil, "The Bayesian approach applied to GPS ambiguity resolution. A mixture model for the discrete-real ambiguities alternative," J. Geodesy, vol. 76, pp. 82-94, 2002.

[16] B. Gundlich and K. Koch, "Confidence regions for GPS baselines by Bayesian statistics," J. Geodesy, vol. 76, pp. 55-62, 2002.

[17] B. Gundlich and P. Teunissen, "Multiple models-fixed, switching, interacting," in V Hotine-Marussi Symposium on Mathematical Geodesy. Berlin, Germany: Springer, 2004, pp. 129-136.

[18] S. Kay, Fundamentals of Statistical Signal Processing: Estimation Theory. , Upper Saddle River, New Jersey, USA: Prentice Hall, 1993.

[19] J. Garcia, P. Roncagliolo, and C. Muravchik, "A novel Bayesian ambiguity resolution technique for GNSS high precision positioning," in Proc. IEEE/ION Position Location and Navigation Symposium (PLANS), 2012, April 2012, pp. 692-699.

[20] K. Koch, Bayesian Inference With Geodetic Applications. Berlin, Germany: Springer, 2007.

[21] Y. Ho and R. Lee, Introduction to Bayesian Statistics. Berlin, Germany: Springer Science \& Business Media, 2007.

[22] Y. Ho and R. Lee, "A Bayesian approach to problems in stochastic estimation and control," IEEE Trans. Autom. Control, vol. 9, no. 4, pp. 333-339, Oct. 1964.

[23] C. D. Burnside, Electromagnetic Distance Measurement. London: Crosby Lockwood Staples, 1974.

[24] H. Blom and Y. Bar-Shalom, "The interacting multiple model algorithm for systems with Markovian switching coefficients," IEEE Trans. Autom. Control, vol. 33, pp. 780-783, 1988.

[25] M. Arulampalam, S. Maskell, N. Gordon, and T. Clapp, "A tutorial on particle filters for online nonlinear/non-Gaussian Bayesian tracking," IEEE Trans. Signal Process., vol. 50, no. 2, pp. 174-188, Feb. 2002.

[26] B. Anderson and J. Moore, Optimal filtering. Upper Saddle River, NJ: Prentice Hall, 1979.

[27] S. Verhagen and P. Teunissen, "New global navigation satellite system smbiguity resolution method compared to existing approaches," $J$. Guidance, Control, and Dynamics, vol. 29, no. 4, pp. 981-991, 2006.

[28] P. Teunissen, "Penalized GNSS ambiguity resolution," J. Geodesy, vol. 78, no. 4-5, pp. 235-244, 2004.

[29] E. Kaplan and C. Hegarty, Understanding GPS: Principles and Applications. Norwood, MA: Artech House, 2006.

[30] J. Garcia, P. Mercader, and C. Muravchik, "Use of GPS carrier phase double differences," Latin Amer. Appl. Res., vol. 33, no. 2, pp. 115-120, 2005.

[31] P. Roncagliolo, J. Garcia, P. Mercader, D. Fuhrmann, and C. Muravchik, "Maximum-likelihood attitude estimation using GPS signals," Digital Signal Process., vol. 17, no. 6, pp. 1089-1100, 2007.

[32] P. Teunissen, "The least-squares ambiguity decorrelation adjustment: A method for fast GPS integer ambiguity estimation," J. Geodesy, vol. 70, no. 1-2, pp. 65-83, 1995.

[33] P. De Jonge and C. Tiberius, "A canonical theory for short GPS baselines. Part III: The geometry of the ambiguity search space," $J$. Geodesy, vol. 71, no. 8, pp. 486-501, 1997.
[34] P. De Jonge and C. Tiberius, "LAMBDA method for integer ambiguity estimation: Implementation aspects," Delft Geodetic Computing Centre LGR Series, no. 12, 1996.

[35] S. Verhagen and B. Li, LAMBDA-Matlab Implementation, version 3.0. Delft, Netherlands: Delft University of Technology and Curtin University, 2012.

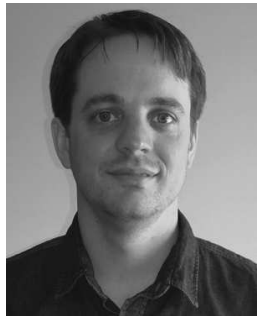

Javier G. Garcia received the Electronics Engineering degree from the National University of La Plata (UNLP), Argentina, in 2003. He is currently a Professor in the UNLP, involved in research and development of Global Navigation Satellite Systems (GNSS) receivers for aerospace applications. His interests are in Statistical Signal Processing, Digital Communications, and Embedded Systems with applications to GNSS. He is a member of the IEEE.

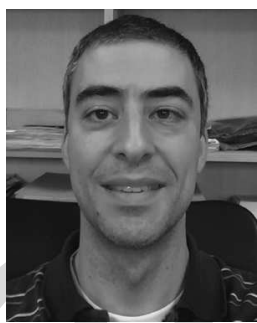

Pedro A. Roncagliolo received the Electronics Engineering and the Ph.D. in Engineering degrees from the National University of La Plata (UNLP), Argentina, in 2001 and 2009. Currently, he is a Professor in the UNLP involved in research and development of Global Navigation Satellite Systems (GNSS) receivers for aerospace applications. His research interests are in Statistical Signal Processing and Radio Frequency Electronics with applications to Wireless Communications and GNSS. He is a member of the IEEE.

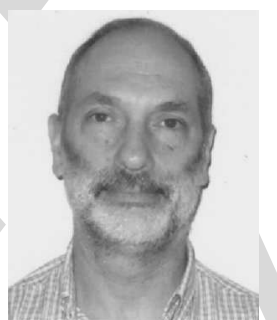

Carlos H. Muravchik (S'81-M'83-SM'99) graduated as an Electronics Engineer from the National University of La Plata, Argentina, in 1973. He received the M.Sc. in Statistics (1983) and the M.Sc. (1980) and Ph.D. (1983) degrees in Electrical Engineering from Stanford University, Stanford, CA.

$\mathrm{He}$ is a Professor at the Department of Electrical Engineering in the National University of La Plata (Argentina); the past director of its Industrial Electronics, Control and Instrumentation Laboratory (LEICI) and a member of the Comision de Investigaciones Cientificas de la Pcia. de Buenos Aires. He was a Visiting Professor to Yale University in 1983 and 1994, to the University of Illinois at Chicago in 1996, 1997, 1999, and 2003 and to Washington University in St Louis in 2006 and 2010. Since 1999 he is a member of the Advisory Board of the journal Latin American Applied Research and was an Associate Editor of the IEEE Transactions on Signal Processing (2003-2006). His research interests are in the area of statistical and array signal processing with biomedical, communications and control applications, and in nonlinear control systems. He is a senior member of the IEEE. 